\title{
MEAN-FIELD MAGNETOHYDRODYNAMICS
}

\section{AS A BASIS OF SOLAR DYNAMO THEORY}

\author{
K.-H. RÄDLER \\ Zentralinstitut für Astrophysik, Potsdam, G.D.R.
}

\section{Introduction}

One of the most striking features of both the magnetic field and the motions observed at the Sun is their highly irregular or random character which indicates the presence of rather complicated magnetohydrodynamic processes. Of great importance in this context is a comprehension of the behaviour of the large scale components of the magnetic field; large scales are understood here as length scales in the order of the solar radius and time scales of a few years. Since there is a strong relationship between these components and the solar 22-years cycle, an insight into the mechanism controlling these components also provides for an insight into the mechanism of the cycle. The large scale components of the magnetic field are determined not only by their interaction with the large scale components of motion. On the contrary, a very important part is played also by an interaction between the large and the small scale components of magnetic field and motion so that a very complicated situation has to be considered.

There have been a number of investigations on this within the framework of meanfield magnetohydrodynamics. This theory developed for magnetohydrodynamic phenomena with irregular character supposes that each of the electromagnetic and hydrodynamic fields is understood as a superposition of a mean and a fluctuating field. The mean fields are defined by taking suitable averages over the original fields and correspond, except for some restrictions, to the large scale fields explained above. From the usual equations of magnetohydrodynamics, equations are deduced governing the behaviour of mean fields in the presence of fluctuations. On this basis a dynamo theory of the solar cycle has been developed which reflects and explains many of the observed features and, in addition, allows conclusions on parameters relating to processes in layers inaccessible for observations.

In this paper a survey will be given on the fundamentals and some special results of mean-field magnetohydrodynamics as far as they are of interest for the dynamo theory of the solar cycle. The representation of mean-field magnetohydrodynamics is closely related to that in the foregoing papers by Krause (see pp. 305-321). As for the dynamo theory itself, only some more general statements will be made. Results obtained for special dynamo models of the solar cycle will be summarized and discussed in the following paper by Stix (see pp. 367-388).

The central and furthest developed part of mean-field magnetohydrodynamics is the mean-field electrodynamics dealing with the question of how mean electromagnetic fields are influenced by the motion of the matter but considering this motion as given. To begin with, we shall be concerned with mean-field electrodynamics and only then include the influence of the electromagnetic fields on the motion, thus arriving at mean-field magnetohydrodynamics per se. 


\section{Mean Field Electrodynamics}

\subsection{BASIC FEATURES}

The mean field electrodynamics, as a deductive theory, has been initiated by Steenbeck et al. (1963, 1966; Rädler, 1966; Krause, 1967) and elaborated in manifold respect by several authors; for summarizing representations see Krause and Rädler (1971), Roberts (1971), Roberts and Stix (1971), Roberts and Soward (1975). To represent the basic features of this theory we turn our attention to the electromagnetic fields in electrically conducting matter carrying out motions which are considered to be given. The behaviour of these electromagnetic fields is governed by Maxwell's and the corresponding constitutive equations. Accepting the suppositions usual in magnetohydrodynamics we have

$$
\begin{aligned}
& \operatorname{curl} \mathbf{E}=-\frac{\partial \mathbf{B}}{\partial t} \quad \operatorname{curl} \mathbf{H}=\mathbf{j} \quad \operatorname{div} \mathbf{B}=0 \\
& \mathbf{B}=\mu \mathbf{H} \quad \mathbf{j}=\sigma(\mathbf{E}+\mathbf{u} \times \mathbf{B}) .
\end{aligned}
$$

Here $\mathbf{B}$ and $\mathbf{H}$ are magnetic flux density and field strength, $\mathbf{E}$ and $\mathbf{j}$ electric field strength and current density, $\mu$ and $\sigma$ the vacuum permeability and the electric conductivity of the matter, and $u$ the velocity of the matter. The equations (2.1) allow the determination of $\mathbf{B}, \mathbf{H}, \mathbf{E}$, and $\mathbf{j}$ if, apart from initial or boundary conditions, $\mathbf{u}$ is given. As is well known Equations (2.1) can be reduced to

$$
\frac{1}{\mu \sigma} \Delta \mathbf{B}+\operatorname{curl}(\mathbf{u} \times \mathbf{B})-\frac{\partial \mathbf{B}}{\partial t}=0 \quad \operatorname{div} \mathbf{B}=0 .
$$

If then $\mathbf{B}$ and $\mathbf{u}$ are known, from (2.1) we get immediately $\mathbf{H}, \mathbf{E}$, and $\mathbf{j}$.

With respect to situations in which the electromagnetic fields and the velocity field show an irregular character we split each of them into a mean and fluctuating part. The mean fields are understood as averages of the original ones. The average of a quantity, say $F$, is denoted by $\bar{F}$, and $F-\bar{F}$ by $F^{\prime}$, so that $F=\bar{F}+F^{\prime}$. The special definition of the averaging procedure is unimportant. It has only to ensure that Reynolds' averaging rules as well as the commutation rule for averaging and derivations or integrations hold. From the theoretical point of view ensemble averages are to be preferred for which these rules are clearly justified. In order to avoid difficulties in comparing theoretical results with observations, however, averages taken over certain ranges in space or time should be considered. In this case Reynolds' rules are solely approximations for situations in which the averaged quantities vary only weakly within each of these ranges. As before the commutation rule can easily be justified here.

We now particularize

$$
\mathbf{B}=\overline{\mathbf{B}}+\mathbf{B}^{\prime} \quad \mathbf{u}=\overline{\mathbf{u}}+\mathbf{u}^{\prime}
$$

with the mean magnetic flux density and the mean velocity, $\overline{\mathbf{B}}$ and $\overline{\mathbf{u}}$, as well as the fluctuations of magnetic flux density and velocity, $\mathbf{B}^{\prime}$ and $\mathbf{u}^{\prime}$. In most of the cases of interest fluctuations as described by $\mathbf{B}^{\prime}$ and $\mathbf{u}^{\prime}$ are specified to represent a turbulence. 
But the following general considerations apply also in cases where fluctuations with certain regular features, e.g. periodicity in space or time, occur.

The objective of the mean field electrodynamics is the determination of the mean electromagnetic fields, $\overline{\mathbf{B}}, \overline{\mathbf{H}}, \overline{\mathbf{E}}$, and $\overline{\mathbf{j}}$, if the mean velocity field, $\overline{\mathbf{u}}$, and some properties of the fluctuating part of the velocity field, $\mathbf{u}^{\prime}$, are given. Taking the average of Equations (2.1), with regard to (2.3) we get

$$
\begin{aligned}
& \operatorname{curl} \overline{\mathbf{E}}=-\frac{\partial \overline{\mathbf{B}}}{\partial t} \quad \operatorname{curl} \overline{\mathbf{H}}=\overline{\mathbf{j}} \quad \operatorname{div} \overline{\mathbf{B}}=0 \\
& \overline{\mathbf{B}}=\mu \overline{\mathbf{H}} \quad \overline{\mathbf{j}}=\sigma\left(\mathbf{E}+\overline{\mathbf{u}} \times \overline{\mathbf{B}}+\overline{\mathbf{u}^{\prime} \times \mathbf{B}^{\prime}}\right) .
\end{aligned}
$$

The formal correspondence between the basic Equations (2.1) and (2.4) for the original and the mean fields is disturbed only by Ohm's law, i.e. (2.1e) and (2.4e), where in the case of mean fields an additional electromotive force, $\overline{\mathbf{u}^{\prime} \times \mathbf{B}^{\prime}}$, appears. In order to determine the mean electromagnetic fields, $\overline{\mathbf{B}}, \overline{\mathbf{H}}, \overline{\mathbf{E}}$, and $\overline{\mathbf{j}}$, in addition to the mean velocity, $\overline{\mathbf{u}}$, this electromotive force $\overline{\mathbf{u}^{\prime} \times \mathbf{B}^{\prime}}$, has to be known.

Basically, by means of Equations (2.2) and (2.3) the quantity $\mathbf{B}^{\prime}$ can be represented as a functional of $\overline{\mathbf{u}}, \mathbf{u}^{\prime}$, and $\overline{\mathbf{B}}$. Consequently, the quantity $\overline{\mathbf{u}^{\prime} \times \mathbf{B}^{\prime}}$ is also to be considered as a functional of $\overline{\mathbf{u}}, \mathbf{u}^{\prime}$, and $\overline{\mathbf{B}}$. After replacing $\overline{\mathbf{u}^{\prime} \times \mathbf{B}^{\prime}}$ by an expression of this kind, Equations (2.4) allow the determination of $\overline{\mathbf{B}}, \overline{\mathbf{H}}, \overline{\mathbf{E}}$, and $\overline{\mathbf{j}}$ from $\overline{\mathbf{u}}$ and quantities depending on $\mathbf{u}^{\prime}$.

Hence, the crucial point of mean-field electrodynamics is the investigation of the electromotive force $\overline{\mathbf{u}^{\prime} \times \mathbf{B}^{\prime}}$. Fortunately some interesting conclusions on $\overline{\mathbf{u}^{\prime} \times \mathbf{B}^{\prime}}$ may be drawn even without detailed calculations. From Equations (2.2) and (2.3) follows that $\overline{\mathbf{u}^{\prime} \times \mathbf{B}^{\prime}}$, regarded as functional of $\overline{\mathbf{u}}, \mathbf{u}^{\prime}$, and $\overline{\mathbf{B}}$, is linear with respect to $\overline{\mathbf{B}}$. Following the usual notation we therefore write

$$
\mathscr{E}=\overline{\boldsymbol{u}^{\prime} \times \bar{B}^{\prime}}=\mathscr{E}^{B}+\mathscr{\mathscr { E }}^{0}
$$

where $\mathscr{E}^{B}$ means a linear homogeneous functional of $\overline{\mathbf{B}}$, and $\mathscr{E}^{0}$ a quantity independent of $\overline{\mathbf{B}}$. Obviously, $\mathscr{E}^{B}$ can be represented by

$$
\mathscr{E}_{i}^{B}(\mathbf{x}, t)=\iint_{0}^{\infty} K_{i j}(\mathbf{x}, t ; \xi, \tau) \bar{B}_{j}(\mathbf{x}-\xi, t-\tau) \mathrm{d} \xi \mathrm{d} \tau
$$

with $K_{i j}$ depending on $\overline{\mathbf{u}}$ and $\mathbf{u}^{\prime}$ but not on $\overline{\mathbf{B}}$. Here as well as in the following the $\boldsymbol{\xi}$-integration is over all space. Since $\boldsymbol{B}^{B}$ for a given time may not depend on $\overline{\mathbf{B}}$ at subsequent times the $\tau$-integration is restricted to $\tau \geq 0$. As far as $\mathscr{E}^{0}$ is concerned we shall just note that it is zero at least as long as the assumption is justified that $\mathbf{B}^{\prime}$ vanishes if $\overline{\mathbf{B}}$ is zero.

It is to be expected that fluctuating quantities as $\mathbf{u}^{\prime}$ and $\mathbf{B}^{\prime}$ at a given point in space and time show no correlation with any quantities at any points far away from the considered one. Consequently, $\boldsymbol{E}^{B}$ for a given point depends only on the behaviour of $\overline{\mathbf{u}}, \mathbf{u}^{\prime}$, and $\overline{\mathbf{B}}$ in confined surroundings of it defined by certain characteristic lengths and times. For the determination of $K_{i j}$ for given $\mathbf{x}$ and $t$, therefore, $\overline{\mathbf{u}}$ and $\mathbf{u}^{\prime}$ are 
needed for such surroundings only, and $K_{i j}$ can be regarded as zero if $\xi$ and $\tau$ considerably exceed the characteristic lengths and times.

We now assume that $\overline{\mathbf{B}}$ varies only weakly in space and time so that its behaviour in the respective surroundings of a given point is already determined by $\overline{\mathbf{B}}$ itself and a few of its derivatives in this point. Then $\mathscr{E}^{B}$ can be represented as a function of $\overline{\mathbf{B}}$ and its derivatives in the point considered. In the simple case in which no other than the first spatial derivatives are included we have

$$
\mathscr{C}_{i}^{B}=a_{i j} \bar{B}_{j}+b_{i j k} \frac{\partial \bar{B}_{j}}{\partial x_{k}}
$$

where the coefficients $a_{i j}$ and $b_{i j k}$ are averaged quantities depending on $\overline{\mathbf{u}}$ and $\mathbf{u}^{\prime}$. Clearly it holds

$$
a_{i j}=\iint_{0}^{\infty} K_{i j}(\mathbf{x}, t ; \xi, \tau) \mathrm{d} \xi \mathrm{d} \tau \quad b_{i j k}=-\iint_{0}^{\infty} K_{i j}(\mathbf{x}, t ; \xi, \tau) \xi_{k} \mathrm{~d} \xi \mathrm{d} \tau
$$

The complete determination of $\boldsymbol{E}^{B}$ requires the knowledge of $K_{i j}$. Fortunately some far-reaching conclusions on $\mathscr{E}^{B}$ can be drawn, even without using $K_{i j}$, from special suppositions on $\overline{\mathbf{u}}$ and the statistical properties of $\mathbf{u}^{\prime}$. In this way expressions for $\mathscr{E}^{B}$ can be given for special situations of interest, and only some factors remain to be determined. This will be illustrated below.

Departing from Equations (2.2) and (2.3) a method was developed for the complete determination of $\boldsymbol{E}^{B}$ which especially provides for explicit expressions of $K_{i j}$. In an approximation in which only second order correlations of $\mathbf{u}^{\prime}$ are considered and all higher ones are neglected it is found

$$
K_{i j}(\mathbf{x}, t ; \xi, \tau)=\varepsilon_{i k l} \varepsilon_{m n p} \varepsilon_{p q j} \frac{\partial G_{l m}(\mathbf{x}, t ; \mathbf{x}-\xi, t-\tau)}{\partial \xi_{n}} Q_{k q}(\mathbf{x}, t ;-\xi,-\tau) .
$$

Here $G_{i j}$ denotes Green's functions of Equation (2.2a) with $\mathbf{u}=\overline{\mathbf{u}}$. In the simplest case, $\overline{\mathbf{u}}=0$, one has

$$
\begin{aligned}
& G_{i j}(\mathbf{x}, t ; \xi, \tau)=\delta_{i j} G(|\mathbf{x}-\xi|, t-\tau) \\
& G(x, t)=\left(\frac{\mu \sigma}{4 \pi t}\right)^{3 / 2} \exp \left\{-\frac{\mu \sigma x^{2}}{4 t}\right\} .
\end{aligned}
$$

Furthermore, $Q_{i j}$ is the second order correlation tensor of $\mathbf{u}^{\prime}$ defined by

$$
Q_{i j}(\mathbf{x}, t ; \xi, \tau)=\overline{u_{i}^{\prime}(\mathbf{x}, t) u_{j}^{\prime}(\mathbf{x}+\xi, t+\tau)} .
$$

The approximation can be improved by adding expressions with higher order correlation tensors to the right-hand side of Equation (2.9).

\subsection{Illustrative EXAMPLE}

As a first example mostly the case is discussed in which the motion is supposed to have no mean part, $\overline{\mathbf{u}}=0$, but to consist of fluctuations, $\mathbf{u}^{\prime}$, representing a homogene- 
ous isotropic turbulence. Homogeneity and isotropy mean that all averaged quantities depending on the $\mathbf{u}^{\prime}$ field are invariant against arbitrary translations of this field and against arbitrary rotations of it around arbitrary axes. Returning to Equation (2.7) for $\boldsymbol{E}^{B}$ and bearing in mind that this especially holds for the coefficients $a_{i j}$ and $b_{i j k}$ we conclude that they are constant in space and have the structures $\alpha \delta_{i j}$ and $\beta \varepsilon_{i j k}$ so that

$$
\mathscr{E}^{B}=\alpha \overline{\mathbf{B}}-\beta \operatorname{curl} \overline{\mathbf{B}} .
$$

Obviously, $\alpha$ is a pseudoscalar but $\beta$ a scalar, both depending on $\mathbf{u}^{\prime}$. With respect to $\mathscr{\mathscr { C }}^{0}$ we furthermore suppose that, if another excitation of $\mathbf{B}^{\prime}$ than that due to $\overline{\mathbf{B}}$ exists at all, the seeds for $\mathbf{B}^{\prime}$ are homogeneously and isotropically distributed. Then we have to conclude that $\mathscr{8}^{0}=0$. In this way Ohm's law (2.4e) becomes

$$
\overline{\mathbf{j}}=\sigma_{T}(\overline{\mathbf{E}}+\alpha \overline{\mathbf{B}})
$$

with

$$
\sigma_{T}=\frac{\sigma}{1+\mu \sigma \beta} .
$$

A homogeneous isotropic turbulence will always be mirror-symmetric too. In this context, mirror-symmetry is defined by the invariance of all averaged quantities depending on the $\mathbf{u}^{\prime}$ field against reflexions of it on arbitrary planes. Since the reflexion of a right-handed screw in the flow pattern produces a left-handed one and vice versa, mirror-symmetry means equipartition between right- and left-handed helical motions.

For a homogeneous isotropic and mirror-symmetric turbulence the pseudoscalar $\alpha$ turns out to be zero. Then the total influence of turbulence on the mean fields can be comprehended as a 'turbulent' conductivity, $\sigma_{T}$, different from the original one, $\sigma$. This statement is related to conceptions like that of eddy viscosity or turbulent diffusion in hydrodynamics. The introduction of a turbulent conductivity has already been proposed by Sweet (1950) and Csada (1951).

For illustration let us consider the electrical current between two assumed electrodes with a given potential difference. At first assuming the medium to be at rest we envisage a current tube, for which an electrical resistance can be defined. If the medium moves, this tube is generally stretched and narrowed so that the resistance increases. The pattern of the mean current, however, coincides with that of the current occurring in the case of the medium at rest. This picture shows clearly that there is a difference between $\sigma_{T}$ and $\sigma$, and we can expect that $\sigma_{T}<\sigma$; see also Steenbeck et al. (1963). As long as the latter relation holds the turbulence enhances the decay of the mean magnetic field.

The value of the turbulent conductivity, $\sigma_{T}$, depends on the coefficient $\beta$. In the second-order correlation approximation we get

$$
\beta=-\frac{1}{3} \iint_{0}^{\infty} \frac{\partial G(\xi, \tau)}{\partial \xi} f(\xi,-\tau) \xi \mathrm{d} \boldsymbol{\xi} \mathrm{d} \tau .
$$


Here $f$ is a longitudinal correlation function defined by

$$
f(\boldsymbol{\xi}, \tau)=\overline{\left(\mathbf{u}^{\prime}(\mathbf{x}, t) \cdot \boldsymbol{\xi}\right)\left(\mathbf{u}^{\prime}(\mathbf{x}+\boldsymbol{\xi}, t+\tau) \cdot \boldsymbol{\xi}\right)} / \xi^{2} .
$$

Incidentally, since isotropy is supposed here, $f$ does not depend on the direction of $\xi$, but on $\xi$ and $\tau$ only. Special results for $\sigma_{T}$, all restricted to second-order correlation approximation, has been given by Rädler $(1966,1968 b)$. Departing from (2.15) and (2.16) the relation $\sigma_{T}<\sigma$ can be confirmed for a wide range of suppositions; see Krause and Roberts (1973a, b), Roberts and Soward (1975). A comparison between theoretical results on $\sigma_{T}$ and observational data of the Sun is given by Krause (1975).

Although the case of a homogeneous isotropic turbulence lacking mirrorsymmetry has to be considered as unrealistic it is instructive to be studied. In this way a point can be easily seen which is important with respect to more complicated kinds of turbulence as to be observed at rotating bodies. There really is a lack of mirror-symmetry, i.e. a predominance of either right-handed or left-handed helical motions.

For a homogeneous isotropic non-mirror-symmetric turbulence the coefficient $\alpha$ is no longer zero. Then an additional electromotive force, $\alpha \overline{\mathbf{B}}$, has to be taken into account which is parallel or antiparallel to the mean magnetic flux. The occurrence of this electromotive force is called ' $\alpha$-effect'. Within the framework of a deductive theory, it has been first discussed by Steenbeck et al. (1966); it should be noted, however, that it was already involved in ideas expressed by Parker (1955).

The $\alpha$-effect becomes plausible if we consider the deformation of originally straight magnetic flux tubes by helical motions. Due to the magnetic field being at least partially frozen in the medium it is clear that situations as those in Figure 1 occur. Roughly speaking, in addition to the originally straight flux tubes, annular flux
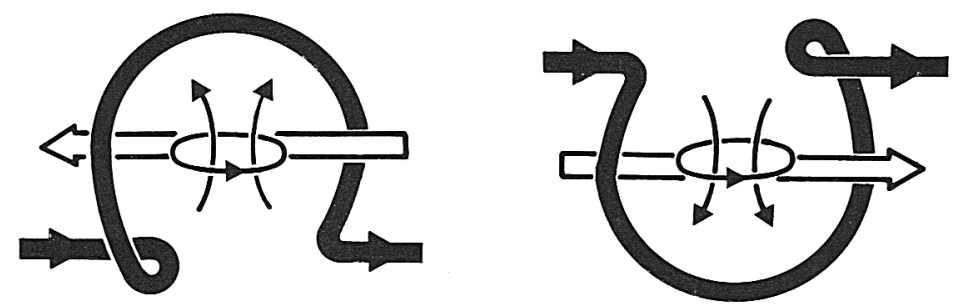

Fig. 1. Deformation of originally straight magnetic flux tubes by helical motions. The magnetic flux tubes are represented by the heavy lines, the electric currents are indicated by arrows, and the motions by the thin lines.

tubes result, which are accompanied by currents crossing the latters' planes. Since these planes do not coincide with the plane of the drawn figure there are components of these currents parallel or antiparallel to the original magnetic flux. If there is an equipartition between right- and left-handed helical motions we have also an equipartition between components parallel and antiparallel to the original flux and, therefore, on average no current. However, if this equipartition is disturbed we have on average a current parallel or antiparallel to the magnetic flux depending on whether left- or right-handed helical motions predominate. 
In the second-order correlation approximation we have

$$
\begin{aligned}
\alpha & =-\frac{1}{3} \iint_{0}^{\infty} \frac{\partial G(\xi, \tau}{\partial \xi} h(\xi,-\tau) \mathrm{d} \xi \mathrm{d} \tau \\
& =-\frac{1}{3} \iint_{0}^{\infty} G(\xi, \tau) h^{*}(\xi,-\tau) \mathrm{d} \xi \mathrm{d} \tau
\end{aligned}
$$

with correlation functions, $h$ and $h^{*}$, defined by

$$
\begin{aligned}
& h(\xi, \tau)=\overline{\left(\mathbf{u}^{\prime}(\mathbf{x}, t) \times \mathbf{u}^{\prime}(\mathbf{x}+\xi, t+\tau)\right)} \cdot \boldsymbol{\xi} / \xi \\
& h^{*}(\xi, \tau)=\overline{\mathbf{u}^{\prime}(\mathbf{x}, t) \cdot \operatorname{curl} \mathbf{u}^{\prime}(\mathbf{x}+\xi, t+\tau)}
\end{aligned}
$$

Obviously, $h$ and $h^{*}$ are measures for the occurrence of helical motions. As proposed by Moffatt $(1970 \mathrm{a}), h^{*}(0,0)$ is called 'helicity'. Further, $h^{*}$ is positive or negative if right or left-handed helical motions predominate. Accordingly, $\alpha$ is positive or negative and, consequently, $\alpha \overline{\mathbf{B}}$ parallel or antiparallel to $\overline{\mathbf{B}}$ if we have preferably left- or right-handed motions, respectively.

The most important feature of the $\alpha$-effect is that it allows the regeneration and the growth of mean magnetic fields. In order to demonstrate this let us consider the situation in an infinitely extended medium where the mean fields satisfy the Equations (2.4) with Ohm's law as specified by (2.13). Then we have the equations

$$
\frac{1}{\mu \sigma_{T}} \Delta \overline{\mathbf{B}}+\alpha \operatorname{curl} \overline{\mathbf{B}}-\frac{\partial \overline{\mathbf{B}}}{\partial t}=0 \quad \operatorname{div} \overline{\mathbf{B}}=0
$$

allowing solutions

$$
\overline{\mathbf{B}}=\left(\mathbf{C} \cos (\mathbf{k} \cdot \mathbf{x}) \pm\left(\mathbf{C} \times \frac{\mathbf{k}}{k}\right) \sin (\mathbf{k} \cdot \mathbf{x})\right) \exp \left\{-\left(\frac{k^{2}}{\mu \sigma_{T}} \mp \alpha k\right) t\right)
$$

with arbitrary vectors $\mathbf{C}$ and $\mathbf{k}$ satisfying $\mathbf{C} \cdot \mathbf{k}=0$. Obviously, such $\overline{\mathbf{B}}$ modes are stationary if $\mu \sigma_{T} \alpha / k= \pm 1$, and there are always $\overline{\mathbf{B}}$ modes which grow with time if $\mu \sigma_{T}|\alpha| / k>1$.

\subsection{REMARKS ON THE APPLICABILITY TO THE SUN}

If we want to apply this general conception to conceive electromagnetic phenomena at the Sun, especially in its convection zone, some points have to be reconsidered.

The first point concerns the split-up of fields into mean fields and fluctuations defined on the basis of an averaging procedure. By reasons already mentioned, averages over space or time are to be considered. Furthermore, the averaging procedure should be compatible with the conception used in all previous work too that, e.g., differential rotation is considered as mean motion whereas all convection occurs as fluctuation. Finally, the validity of Reynolds rules supposed above has to be ensured. As mentioned also by Krause (1975) and Stix (1975), averages taken over a 
spatial volume hardly show the properties required. Such averages cannot even separate differential rotation and convection as far as large convection cells are considered; the reason is that the orders of length scales of the two do not clearly differ. A similar difficulty arises with the magnetic fields. A way out could be to replace the average over a volume by an average over longitude as used by Braginski (1964). Then all requirements mentioned are fulfilled automatically. But we have to put up with the restriction to axisymmetric mean fields; all non-axisymmetric parts of the original fields occur as fluctuations. Following Stix (1975) again we consider it best to use averages over a time span in the order of about one or two years. In this case the requirements formulated above are satisfied at least in a certain approximation. This is due to the fact that the spectrum of characteristic times observed at the Sun shows a gap for times in the order of one or two years.

For the next point to be discussed the magnetic Reynolds number, $R_{m}$, and the Strouhal number, $S$, both in relation to fluctuations, are of interest. They are defined by

$$
R_{m}=\mu \sigma u_{c}^{\prime} \lambda_{c} \quad S=u_{c}^{\prime} \tau_{c} / \lambda_{c}
$$

where $u_{c}^{\prime}$ represents a characteristic value of the fluctuating velocity, e.g. $\sqrt{\overline{u^{\prime 2}}}$, and $\lambda_{c}$ and $\tau_{c}$ are characteristic length and time, e.g., correlation length and time. It holds $\mu=4 \pi \times 10^{-7} \mathrm{Vs} \mathrm{A}^{-1} \mathrm{~m}^{-1}$, and we accept $\sigma=10^{3} \Omega^{-1} \mathrm{~m}^{-1}$. With respect to granules we take $u_{c}^{\prime}=2 \times 10^{3} \mathrm{~m} \mathrm{~s}^{-1}, \lambda_{c}=2 \times 10^{6} \mathrm{~m}$, and $\tau_{c}=3 \times 10^{2} \mathrm{~s}$. Then we have $R_{m} \approx$ $5 \times 10^{6}$ and $S \approx 3 \times 10^{-1}$. If we consider supergranules and choose $u_{c}^{\prime}=4 \times 10^{2} \mathrm{~m} \mathrm{~s}^{-1}$, $\lambda_{c}=3 \times 10^{7} \mathrm{~m}$, and $\tau_{c}=5 \times 10^{4} \mathrm{~s}$ it results $R_{m} \approx 2 \times 10^{7}$ and $S \approx 7 \times 10^{-1}$.

We reconsider now the transition from the general to the special expression of $\boldsymbol{E}^{B}$ given by (2.6) and (2.7) and ask whether other derivatives of $\overline{\mathbf{B}}$ have to be included. As mentioned above, $K_{i j}$ can be regarded to be zero if $\xi$ and $\tau$ considerably exceed certain characteristic values, say $\lambda_{K}$ and $\tau_{K}$. From the expressions of $K_{i j}$ it can easily be read that

$$
\begin{aligned}
& \lambda_{K}=\lambda_{c} \quad \tau_{K}=\mu \sigma \lambda_{c}^{2} \quad \text { for } R_{m} / S \ll 1 \\
& \lambda_{K}=\sqrt{\tau_{c} / \mu \sigma} \quad \tau_{K}=\tau_{c} \quad \text { for } R_{m} / S \gg 1 .
\end{aligned}
$$

The contributions to $\boldsymbol{\delta}^{B}$ due to higher derivatives of $\overline{\mathbf{B}}$ are, e.g., in the order of $\tau_{K}(\partial \overline{\mathbf{B}} / \partial t)$ or $\lambda_{K}^{2}\left(\partial^{2} \overline{\mathbf{B}} / \partial x_{i} \partial x_{j}\right)$; see also Rädler (1968b). Since always $R_{m} / S \gg 1$, with the data of granules we have $\lambda_{K} \approx 5 \times 10^{2} \mathrm{~m}$ and $\tau_{K} \approx 3 \times 10^{2} \mathrm{~s}$, with the data of supergranules $\lambda_{K} \approx 6 \times 10^{3} \mathrm{~m}$ and $\tau_{K} \approx 5 \times 10^{4} \mathrm{~s}$. Therefore, those contributions seem to be negligible.

Finally we deal with the validity of the above-mentioned second-order correlation approximation for the coefficients occurring in special expressions of $\boldsymbol{E}^{B}$. This is of interest because higher order approximations require very lengthy calculations which have been done up to now only in a few exceptional cases. A simple sufficient condition for the validity, which can easily be read from the deductions, is

$$
\min \left(R_{m}, S\right) \ll 1 \text {. }
$$

With the data used above this is scarcely fulfilled. It must be noted, however, that (2.23) is not a necessary condition. The values of the turbulent conductivity which 
have been obtained within the frame of the second-order correlation approximation fit very well to observational data; see Krause (1967, 1975), Rädler (1968b), Steenbeck and Krause (1969), Krause and Rüdiger (1975). In this way, there is at least some reason to believe that the results obtained from the approximation are reliable for the solar convection zone.

\subsection{OHM'S LAW FOR THE SOLAR CONVECTION ZONE}

2.4.1. Elaborating the mean-field electrodynamics for the Sun we have to consider that, in contraist to the simple example discussed above, there are firstly a nonvanishing mean motion, at least the differential rotation, and secondly fluctuating motions, like the convection, which can no longer be treated as a homogeneous isotropic turbulence. Already the intensity of these fluctuating motions shows a radial dependence, and there are significant differences between the velocity components in radial and horizontal directions. In addition, these motions are subjected to Coriolis forces. Taking all this into account, the homogeneity is disturbed, and instead of isotropy we have to take into consideration that in each point at least two preferred directions exist, namely a radial and an axial one. Finally, there must be deviations from mirror-symmetry because of the preference of these directions.

Studying now Ohm's law for the mean fields we first consider the cases in which only the radial or only the axial direction is preferred and then pass over to the case where both are included. Furthermore, we first deal only with effects described by the electromotive force $\boldsymbol{\delta}^{B}$ in the form given in (2.7), and we neglect $\boldsymbol{\delta}^{0}$ which will be discussed later.

2.4.2. Let us now suppose that isotropy and mirror-symmetry of the fluctuating motions are disturbed only by the preference of the radial direction. More precisely, all averaged quantities depending on the fluctuating velocity field are considered to be invariant under rotation of this field around a given radius and under reflexion of it at planes containing this radius. By similar arguments as used above $\boldsymbol{E}^{B}$ can readily be specified, and Ohm's law for the mean fields becomes

$$
\begin{aligned}
\mathbf{j}=\sigma_{T} & (\overline{\mathbf{E}}+\overline{\mathbf{u}} \times \overline{\mathbf{B}} \\
& -\gamma \hat{\mathbf{g}} \times \overline{\mathbf{B}} \\
& -\gamma_{1} \hat{\mathbf{g}}(\hat{\mathbf{g}} \cdot \operatorname{curl} \overline{\mathbf{B}})-\gamma_{2} \hat{\mathbf{g}} \times(\hat{\mathbf{g}} \cdot \operatorname{grad}) \overline{\mathbf{B}}-\gamma_{3} \hat{\mathbf{g}} \times(\hat{\mathbf{g}} \cdot \operatorname{grad} \overline{\mathbf{B}})
\end{aligned}
$$

where $\hat{\mathbf{g}}$ is the unit vector in radial direction and $(\hat{\mathbf{g}} \cdot \operatorname{grad} \overline{\mathbf{B}})_{i}$ means $\hat{g}_{j} \partial \bar{B}_{j} / \partial x_{i}$. Incidentally, the last three terms within the brackets can be rewritten to be

$$
-\mu\left(\gamma_{1}-\gamma_{2}\right) \hat{\mathbf{g}}(\hat{\mathbf{g}} \overline{\mathbf{j}})-\mu \gamma_{2} \overline{\mathbf{j}}-\left(\gamma_{2}+\gamma_{3}\right) \hat{\mathbf{g}} \times(\hat{\mathbf{g}} \cdot \operatorname{grad} \overline{\mathbf{B}}) .
$$

Again, a turbulent conductivity, $\sigma_{T}$, was introduced for which (2.15) and also (2.16) hold.

Beside the Lorentz force, $\overline{\mathbf{u}} \times \overline{\mathbf{B}}$, an electromotive force, $-\gamma \hat{\mathbf{g}} \times \overline{\mathbf{B}}$, appears where the part of mean velocity, $\overline{\mathbf{u}}$, is played by a vector quantity, $-\gamma \hat{\mathbf{g}}$, antiparallel or parallel to the radial direction. Just as the Lorentz force, this electromotive force corresponds to a transport of magnetic flux. Such transport different from that due to a mean motion is sometimes called 'pumping of magnetic flux.' 
Within the frame of second-order correlation approximation we have

$$
\gamma=-\frac{1}{2} \iint_{0}^{\infty} \frac{\partial G(\xi, \tau)}{\partial \xi}\left(k_{1}(\xi,-\tau)+k_{2}(\xi,-\tau)\right) \mathrm{d} \xi \mathrm{d} \tau
$$

with correlation functions, $k_{1}$ and $k_{2}$, defined by

$$
\begin{aligned}
& k_{1}(\xi, \tau)=\overline{\left(\mathbf{u}^{\prime}(\mathbf{x}, t) \cdot \xi\right)\left(\mathbf{u}^{\prime}(\mathbf{x}+\xi, t+\tau) \cdot \hat{\mathbf{g}}\right)} / \xi \\
& k_{2}(\xi, \tau)=\overline{\left(\mathbf{u}^{\prime}(\mathbf{x}, t) \cdot \hat{\mathbf{g}}\right)(\mathbf{u}(\mathbf{x}+\xi, t+\tau) \cdot \xi)} / \xi .
\end{aligned}
$$

For a turbulence deviating only by a gradient of the mean intensity from a homogeneous isotropic and mirror-symmetric turbulence, more detailed results on the electromotive force under consideration and especially on the coefficient $\gamma$ have been given by Rädler $(1966,1968 b, 1969 a)$. It turns out that there is a tendency to push out the mean magnetic flux from the regions of high turbulence intensity. Therefore, this effect is sometimes denoted as 'turbulent diamagnetism'. A similar situation occurs if the gradient of intensity is replaced by, e.g., a gradient of correlation length; see Rädler (1969a), Krause and Rädler (1971). In a turbulent layer like the solar convection zone a gradient in intensity, the direction of which must change within the layer, pushes the magnetic flux from the inner regions to both boundaries whereas a gradient in correlation length may push it from one boundary to the other. If the fluctuating motions are specified to represent convective cell motions the electromotive force under consideration describes the effect of 'topological pumping of magnetic flux' discussed by Drobyshevski and Yuferev (1974). In this case, a pumping from one boundary to another only occurs for higher than secondorder correlation approximations.

In addition to the electromotive force discussed there are others connected with spatial derivatives of $\overline{\mathbf{B}}$. A part of them may be described by means of an anisotropic turbulent conductivity too. It shows that $(2.27)$ is equivalent to

$$
\overline{\mathbf{j}}=\boldsymbol{\sigma}_{T}\left(\overline{\mathbf{E}}+\overline{\mathbf{u}} \times \overline{\mathbf{B}}-\gamma \hat{\mathbf{g}} \times \overline{\mathbf{B}}-\left(\gamma_{2}+\gamma_{3}\right) \hat{\mathbf{g}} \times(\hat{\mathbf{g}} \cdot \operatorname{grad} \overline{\mathbf{B}})\right.
$$

with a conductivity tensor, $\sigma_{T}$, given by

$$
\sigma_{T j}=\frac{\sigma_{T}}{\left(1+\mu \sigma_{T} \gamma_{1}\right)\left(1+\mu \sigma_{T} \gamma_{2}\right)}\left(\left(1+\mu \sigma_{T} \gamma_{1}\right) \delta_{i j}-\mu \sigma_{T}\left(\gamma_{1}-\gamma_{2}\right) \hat{g}_{i} \hat{g}_{j}\right)
$$

In the second-order correlation approximation we have

$$
\begin{aligned}
& \gamma_{1}=-\frac{1}{6} \iint_{0}^{\infty} \frac{\partial G(\xi, \tau)}{\partial \xi}\left(f(\xi,-\tau) \xi+3 k_{2}(\xi,-\tau)(\hat{\mathbf{g}} \cdot \boldsymbol{\xi})\right) \mathrm{d} \xi \mathrm{d} \tau \\
& \gamma_{2}=\frac{1}{6} \iint_{0}^{\infty} \frac{\partial G(\xi, \tau)}{\partial \xi}\left(2 f(\xi,-\tau) \xi+3\left(k_{1}(\xi,-\tau)+k_{2}(\xi,-\tau)\right)(\hat{\mathbf{g}} \cdot \boldsymbol{\xi})\right) \mathrm{d} \xi \mathrm{d} \tau
\end{aligned}
$$




$$
\gamma_{3}=\frac{1}{6} \iint_{\sigma_{0}}^{\infty} \frac{\partial G(\xi, \tau)}{\partial \xi}\left(f(\xi,-\tau) \xi+3 k_{1}(\xi,-\tau)(\hat{\mathbf{g}} \cdot \boldsymbol{\xi})\right) \mathrm{d} \xi \mathrm{d} \tau
$$

with $f, k_{1}$, and $k_{2}$ as given above. In this context we refer to the results obtained for the extreme case of a homogeneous two-dimensional turbulence with no motion in the preferred direction; see Krause and Rüdiger (1975). They can easily be formulated in terms of an anisotropic turbulent conductivity; in this case $k_{1}$ and $k_{2}$ turn out to be zero. For the above-mentioned turbulence with a gradient of mean intensity, the second-order correlation tensor is linear in this gradient; see Rädler (1966, 1974). Therefore, in the applied approximation $\gamma_{1}, \gamma_{2}$ and $\gamma_{3}$ vanish; see Rädler (1966, 1968b).

2.4.3. Now we pass over to the case in which isotropy and mirror-symmetry of the fluctuating motions are violated only by the influence of Coriolis forces, which occur as a consequence of the rotation. Then the averaged quantities dependent from the fluctuating velocity field have to be invariant under rotation of this field around axes parallel to the rotational axis and under reflexions at planes perpendicular to it. For simplicity we suppose the influence of Coriolis forces as weak enough in order to neglect in the following all quantities of higher than first order with regard to the angular velocity. In the same way as above we get

$$
\overline{\mathbf{j}}=\sigma_{T}\left(\overline{\mathbf{E}}+\overline{\mathbf{u}} \times \overline{\mathbf{B}}-\beta_{1}(\hat{\boldsymbol{\omega}} \cdot \operatorname{grad}) \overline{\mathbf{B}}-\beta_{2} \operatorname{grad}(\hat{\boldsymbol{\omega}} \cdot \overline{\mathbf{B}})\right)
$$

where $\hat{\boldsymbol{\omega}}$ is the unit vector parallel to the rotational axis. If we relinquish linearity in the angular velocity, four other terms have to be added at the right-hand side of this equation. The last two terms within the brackets in (2.31) can be rewritten to be

$$
\mu \beta_{1} \hat{\boldsymbol{\omega}} \times \overline{\mathbf{j}}-\left(\beta_{1}+\beta_{2}\right) \operatorname{grad}(\hat{\boldsymbol{\omega}} \cdot \overline{\mathbf{B}}) .
$$

As before, a turbulent conductivity, $\sigma_{T}$, appears for which (2.15) and (2.16) hold.

The most interesting feature of this result is, however, the occurrence of an electromotive force, $\mu \boldsymbol{\beta}_{1} \hat{\boldsymbol{\omega}} \times \overline{\mathbf{j}}$, often called ' $\boldsymbol{\omega} \times \mathbf{j}$-effect'. It reminds one of the Hall effect. This $\omega \times \mathbf{j}$-effect can be described in terms of an anisotropic turbulent conductivity too. From (2.31) and (2.32) immediately follows

$$
\overline{\mathbf{j}}=\boldsymbol{\sigma}_{T}\left(\overline{\mathbf{E}}+\overline{\mathbf{u}} \times \overline{\mathbf{B}}-\left(\beta_{1}+\beta_{2}\right) \operatorname{grad}(\hat{\boldsymbol{\omega}} \cdot \overline{\mathbf{B}})\right)
$$

with a turbulent conductivity tensor, $\sigma_{T}$, given by

$$
\sigma_{T i j}=\frac{\sigma_{T}}{1+\left(\mu \sigma_{T} \beta_{1}\right)^{2}}\left(\delta_{i j}-\mu \sigma_{T} \beta_{1} \varepsilon_{i j k} \hat{\omega}_{k}+\left(\mu \sigma_{T} \beta_{1}\right)^{2} \hat{\omega}_{i} \hat{\omega}_{k}\right)
$$

Contrary to (2.29) it contains an antisymmetric part. The electromotive force $-\left(\beta_{1}+\beta_{2}\right) \operatorname{grad}(\hat{\boldsymbol{\omega}} \cdot \overline{\mathbf{B}})$ plays a minor part. As far as $\boldsymbol{\beta}_{1}+\boldsymbol{\beta}_{2}$ does not depend on space this force may always be compensated by that part of $\overline{\mathbf{E}}$ which results from space charges.

Just as the $\alpha$-effect occurring with homogeneous isotropic non-mirror-symmetric turbulence, the $\omega \times \mathbf{j}$-effect is also caused by helical motions, but it does not require a 
predominance of right-or left-handed helical motions. A similar illustration as given for the $\alpha$-effect by means of Figure 1 is possible for the $\omega \times \mathbf{j}$-effect too. In this case, the anisotropy of the motions has to be taken into account. Furthermore, due to the gradient in the mean flux density two flux tubes as in Figure 1 may differ in flux density. Therefore the helical motions, despite the equipartition of both types, can produce a mean current.

Within the second-order correlation approximation we have

$$
\begin{array}{r}
\beta_{1}=\frac{1}{10} \iint_{0}^{\infty} \frac{\partial G(\xi, \tau)}{\partial \xi}\left(3 h(\xi,-\tau)(\hat{\boldsymbol{\omega}} \cdot \xi)-l_{1}(\xi,-\tau) \xi\right) \mathrm{d} \xi \mathrm{d} \tau \\
\beta_{2}=-\frac{1}{10} \iint_{0}^{\infty} \frac{\partial G(\xi, \tau)}{\partial \xi}\left(2 h(\xi,-\tau)(\hat{\boldsymbol{\omega}} \cdot \xi)-4 l_{1}(\xi,-\tau) \xi\right. \\
\left.+5 l_{2}(\xi,-\tau) \xi\right) \mathrm{d} \xi \mathrm{d} \tau
\end{array}
$$

where $h$ is defined as above and $l_{1}$ and $l_{2}$ are given by

$$
\begin{aligned}
& l_{1}(\xi, \tau)=\overline{\left(\left(\mathbf{u}^{\prime}(\mathbf{x}, t) \times \mathbf{u}^{\prime}(\mathbf{x}+\xi, t+\tau)\right) \cdot \hat{\boldsymbol{\omega}}\right)} \\
& \left.l_{2}(\boldsymbol{\xi}, \tau)=\overline{\left(\mathbf{u}^{\prime}(\mathbf{x}, t) \cdot \boldsymbol{\xi}\right)\left(\left(\mathbf{u}^{\prime}(\mathbf{x}+\boldsymbol{\xi}, t+\tau) \times \hat{\boldsymbol{\omega}}\right)\right.} \quad \xi\right) / \xi^{2} .
\end{aligned}
$$

For an originally homogeneous isotropic and mirror-symmetric turbulence in an incompressible medium subject to Coriolis forces explicit expressions of $\beta_{1}$ and $\beta_{2}$ have been given by Rädler (1969a, b), and by Roberts and Soward (1975).

2.4.4. Finally, we deal with the situation in which the fluctuating motions are characterized by the simultaneous occurrence of the two preferred directions considered above. Extending the hitherto used conception to this more complicated situation, a rather complex result occurs. Restricting ourselves again to linearity in the angular velocity which is responsible to the Coriolis forces we have

$$
\begin{aligned}
& \overline{\mathbf{j}}=\sigma_{T}(\overline{\mathbf{E}}+\overline{\mathbf{u}} \times \overline{\mathbf{B}} \\
&-\gamma \hat{\mathbf{g}} \times \overline{\mathbf{B}} \\
&-\gamma_{1} \hat{\mathbf{g}}(\hat{\mathbf{g}} \cdot \operatorname{curl} \overline{\mathbf{B}})-\gamma_{2} \hat{\mathbf{g}} \times(\hat{\mathbf{g}} \cdot \operatorname{grad}) \overline{\mathbf{B}}-\gamma_{3} \hat{\mathbf{g}} \times(\hat{\mathbf{g}} \cdot \operatorname{grad} \overline{\mathbf{B}}) \\
&-\beta_{1}(\hat{\boldsymbol{\omega}} \cdot \operatorname{grad}) \overline{\mathbf{B}}-\beta_{2} \operatorname{grad}(\hat{\boldsymbol{\omega}} \cdot \overline{\mathbf{B}}) \\
&-\alpha_{1}(\hat{\mathbf{g}} \cdot \hat{\boldsymbol{\omega}}) \overline{\mathbf{B}}-\alpha_{2}(\hat{\mathbf{g}} \cdot \overline{\mathbf{B}}) \hat{\boldsymbol{\omega}}-\alpha_{3}(\hat{\boldsymbol{\omega}} \cdot \overline{\mathbf{B}}) \hat{\mathbf{g}}-\alpha_{4}(\hat{\mathbf{g}} \cdot \hat{\boldsymbol{\omega}})(\hat{\mathbf{g}} \cdot \overline{\mathbf{B}}) \hat{\mathbf{g}} \\
&-\alpha_{5}(\hat{\mathbf{g}} \cdot \operatorname{grad}(\hat{\boldsymbol{\omega}} \cdot \overline{\mathbf{B}})) \hat{\mathbf{g}}-\alpha_{6}(\hat{\boldsymbol{\omega}} \cdot(\hat{\mathbf{g}} \cdot \operatorname{grad} \overline{\mathbf{B}})) \hat{\mathbf{g}} \\
&-\alpha_{7}(\hat{\mathbf{g}} \cdot \hat{\boldsymbol{\omega}})(\hat{\mathbf{g}} \cdot \operatorname{grad}(\hat{\mathbf{g}} \cdot \overline{\mathbf{B}})) \hat{\mathbf{g}}-\alpha_{8}(\hat{\mathbf{g}} \cdot \operatorname{grad}(\hat{\mathbf{g}} \cdot \overline{\mathbf{B}})) \hat{\boldsymbol{\omega}} \\
&\left.-\alpha_{9}(\hat{\mathbf{g}} \cdot \hat{\boldsymbol{\omega}})(\hat{\mathbf{g}} \cdot \operatorname{grad}) \overline{\mathbf{B}}-\alpha_{10}(\hat{\mathbf{g}} \cdot \hat{\boldsymbol{\omega}})(\hat{\mathbf{g}} \cdot \operatorname{grad} \overline{\mathbf{B}})\right) .
\end{aligned}
$$

Again, other representations are possible too. We especially refer to (2.25) and (2.32), and we add that the terms in the last line can also be written in the form

$$
\mu \alpha_{9}(\hat{\mathbf{g}} \cdot \hat{\boldsymbol{\omega}}) \hat{\mathbf{g}} \times \overline{\mathbf{j}}-\left(\alpha_{9}+\alpha_{10}\right)(\hat{\mathbf{g}} \cdot \hat{\boldsymbol{\omega}})(\hat{\mathbf{g}} \cdot \operatorname{grad} \overline{\mathbf{B}}) .
$$


The electromotive forces in the first four lines of (2.37) are to be expected from the foregoing discussion, and all relations given in this connection apply here too. But there are further terms in which both preferred directions, i.e. $\hat{\mathbf{g}}$ and $\hat{\boldsymbol{\omega}}$, appear simultaneously. In all previous representations of this matter the simplifying assumption of linearity in both $\hat{\mathbf{g}}$ and $\hat{\boldsymbol{\omega}}$ was introduced. In this way, the last term of the fifth line and all following terms did not occur. For a number of reasons this assumption seems rather problematic.

Just as in the case of a homogeneous isotropic and non-mirror-symmetric turbulence we have an electromotive force proportional to the mean magnetic flux density, namely $-\alpha_{1}(\hat{\mathbf{g}} \cdot \hat{\boldsymbol{\omega}}) \overline{\mathbf{B}}$, i.e. an $\alpha$-effect. In contrast to that case, however, this electromotive force is accompanied by the other ones given in the fifth line of (2.37). We shall speak of 'idealized $\alpha$-effect' as long as only $-\alpha_{1}(\hat{\mathbf{g}} \cdot \hat{\boldsymbol{\omega}}) \overline{\mathbf{B}}$ is considered, and of 'real $\alpha$-effect' if the other contributions are included.

In accordance with the $\alpha$-effect occurring with homogeneous isotropic nonmirror-symmetric turbulence, and in contrast to the $\hat{\boldsymbol{\omega}} \times \mathbf{j}$-effect, the $\alpha$-effect considered here is due to the predominance of right- or left-handed helical motions. The illustration given in the case of homogeneous isotropic non-mirror-symmetric turbulence can easily be modified to fit to the situation under discussion.

In the second-order correlation we have

$$
\alpha_{1}(\hat{\mathbf{g}} \cdot \hat{\boldsymbol{\omega}})=\frac{1}{2} \iint_{0}^{\infty} \frac{\partial G(\xi, \tau)}{\partial \xi}\left(h(\xi,-\tau)-m_{1}(\xi,-\tau) \frac{(\hat{\mathbf{g}} \cdot \boldsymbol{\xi})}{\xi}\right) \mathrm{d} \xi \mathrm{d} \tau
$$

with $h$ as defined above and $m_{1}$ given by

$$
m_{1}(\xi, \tau)=\overline{\left(\left(\mathbf{u}^{\prime}(\mathbf{x}, t) \times \mathbf{u}^{\prime}(\mathbf{x}+\xi, t+\tau)\right)\right.} \cdot \hat{\mathbf{g}} .
$$

Due to assumptions introduced here, (2.39) provides for a value of $\alpha_{1}$ independent of $\hat{\mathbf{g}}$ and $\hat{\boldsymbol{\omega}}$. In contrast to the coefficient $\alpha$ given by (2.17), the coefficient $\alpha_{1}$ depends not only on correlations described by the function $h$ or, what is the same, by the function $h^{*}$, but is a more complicated quantity. The corresponding expressions for $\alpha_{2}, \alpha_{3}$, and $\alpha_{4}$, which are rather complicated too, will not be given here.

Departing from various conceptions on the structure of the fluctuating motions several investigations have been carried out which provide for special results on $\alpha_{1}, \alpha_{2}, \ldots$ or related quantities. The motions in the solar convection zone are influenced in a high degree by the stratification of the medium. Investigations which include the effect of stratification were performed by Steenbeck et al. (1966) and by Krause (1967). Furthermore, with other assumptions on the motions, special results were presented by Rädler (1969a), Moffatt (1970a), Krause and Rädler (1971), and Roberts and Soward (1975).

The electromotive forces connected with derivatives of $\overline{\mathbf{B}}$ given in the last three lines of (2.37) have not been investigated in detail up to now.

2.4.5. So far we have taken into account the electromotive force $\boldsymbol{E}^{B}$ only, but have not considered $\mathscr{E}^{0}$ which can be unequal zero under conditions which allow $\mathbf{B}^{\prime}$ to be unequal zero even though $\overline{\mathbf{B}}$ vanishes. Since in the solar convection zone the 
magnetic Reynolds number responsible for fluctuations, $\boldsymbol{R}_{m}$ is much larger than unity we cannot exclude without further investigation that some parts of the fluctuating motion give rise to 'local dynamos', i.e. dynamos restricted to the scales of fluctuations, which contribute to $\mathbf{B}^{\prime}$ also in absence of $\overline{\mathbf{B}}$. This is related to ideas by Batchelor (1950) and Kasantsev (1967). According to the fact that the fluctuating motions vary rapidly, each such dynamo will have a short lifetime only, and the corresponding part of $\mathbf{B}^{\prime}$ excited by some kind of seed will reach only small values and then decay again. Of course, $\boldsymbol{\delta}^{0}$ depends on assumptions about the seeds. For simplicity we suppose the seeds to be isotropically distributed. From the assumptions used above for the fluctuating motions including weak influence of Coriolis forces we can now conclude

$$
\mathscr{E}^{0}=\kappa \hat{\mathbf{g}}+\lambda \hat{\mathbf{g}} \times \hat{\boldsymbol{\omega}}
$$

with some coefficients $\kappa$ and $\lambda$. Taking into account arbitrary strength of Coriolis forces, a term proportional to $\hat{\boldsymbol{\omega}}$ has to be added. Unfortunately there does not exist any investigation which provides for more detailed information on $\boldsymbol{E}^{\circ}$ or coefficients $\kappa$ and $\lambda$.

For the solar cycle we conjecture that the electromotive force $\boldsymbol{\varepsilon}^{B}$, the magnitude of which depends on that of $\overline{\mathbf{B}}$, is always large compared with $\boldsymbol{\varepsilon}^{0}$ so that the latter can be neglected. This seems to follow already from the argument given above in favour of the small efficiency of the local dynamos producing $\mathbf{B}^{\prime}$.

We are faced with a completely different situation when $\overline{\mathbf{B}}$ is zero at a given time. Then $\boldsymbol{E}^{B}$ is zero too, and if the local dynamos lead to a non-vanishing $\boldsymbol{E}^{0}$, we have to expect that $\overline{\mathbf{B}}$ grows. Especially the part of $\mathscr{E}^{0}$ proportional to $\hat{\mathbf{g}} \times \hat{\boldsymbol{\omega}}$ gives rise to a $\overline{\mathbf{B}}$ field of dipole type. Thus, as a consequence of small scale magnetic fields, a largescale field can occur.

\section{Kinematic Dynamo Theory of the Solar Cycle}

\subsection{BASIC FEATURES}

In order to discuss the general principle of the dynamo theory of the solar cycle we consider the Sun as a sphere of electrically conducting matter with a mean motion like differential rotation and with turbulent motions in the outer layers, and we suppose the mean motion as well as the distribution of the turbulence to be symmetric with respect to both the rotation axis and the equatorial plane, and to be stationary. Furthermore, we understand the solar cycle, which should be comprehended theoretically here, substantially as an interplay between a poloidal and a toroidal mean magnetic field, both supposed to be axisymmetric too but antisymmetric with respect to the equatorial plane. In a rough picture, the poloidal field is of dipole type, and the toroidal field consists of two oppositely oriented belts, one in each hemisphere. Both fields generate and attenuate each other so that an oscillation with a period of 22 years results. Compared with the poloidal field the toroidal one reaches much higher amplitudes and is responsible, e.g., for the appearance of sunspots. 
We have first to discuss how a poloidal field can generate a toroidal one and vice versa. Provided a poloidal field exists, a toroidal one must already occur due to differential rotation. Accepting that for the generation of the toroidal field the differential rotation is more effective than the turbulence, we may neglect all effects of turbulence except that represented by the turbulent conductivity. Departing from a toroidal field, however, a poloidal one can be generated neither by differential rotation nor by any other axisymmetric motion but only by turbulence. Parker (1955) was the first to recognize that the poloidal field can occur as a consequence of cyclonic convection. Within the frame of mean-field electrodynamics the effect of cyclonic convection he pointed out is reflected by the $\alpha$-effect.

Let us consider in more detail this crucial point of the dynamo theory of the solar cycle, the generation of a poloidal field from a toroidal one. Having in mind that a poloidal field is connected with toroidal currents we have to ask for electromotive forces caused by turbulence which induce toroidal currents from toroidal fields. Only three of the electromotive forces occurring in (2.37) show this property. With respect to $(2.32)$ and $(2.38)$ we note them in the form

$$
\mu \boldsymbol{\beta}_{1} \hat{\mathbf{\omega}} \times \overline{\mathbf{j}}, \quad-\alpha_{1}(\hat{\mathbf{g}} \cdot \hat{\boldsymbol{\omega}}) \overline{\mathbf{B}}, \quad \mu \alpha_{9}(\hat{\mathbf{g}} \cdot \hat{\boldsymbol{\omega}}) \hat{\mathbf{g}} \times \overline{\mathbf{j}} .
$$

Even if we relinquish linearity in $\hat{\boldsymbol{\omega}}$ no other terms occur here.

In addition to differential rotation at least one of the electromotive forces given in (3.1) is required to have indeed an interaction between poloidal and toroidal magnetic fields. It remains to be scrutinized whether this interaction actually allows a dynamo, i.e., prevents that the magnetic fields vanish in course of time, and whether and under which conditions alternating magnetic fields occur. Unfortunately, the questions arising here can be answered only on the basis of either analytical calculations for extremely simplified models or lengthy numerical calculations for more realistic models.

As the first of the electromotive forces listed in (3.1) we consider that given by $-\alpha_{1}(\hat{\mathbf{g}} \cdot \hat{\mathbf{\omega}}) \overline{\mathbf{B}}$, i.e. the idealized $\alpha$-effect. In conjunction with differential rotation it actually allows dynamos, called $\alpha \omega$-type dynamos, for both stationary and alternating magnetic fields. The dynamo mechanism suggested by Parker (1955) already before the elaboration of mean-field electrodynamics can be understood as being of $\alpha \omega$-type, generating alternating fields; however, he did not investigate spherical dynamo models. Departing from the conception of mean-field electrodynamics Steenbeck and Krause (1969) elaborated spherical dynamo models of the $\alpha \omega$-type for alternating fields. They presented numerical results on excitation conditions, space and time structure of the fields etc. and compared them with observational material as the butterfly diagram. Meanwhile numerous investigations of such models with alternating fields have been carried out; see, e.g., Deinzer and Stix (1971), Roberts and Stix (1972), Roberts (1972), Ruzmaikin and Ivanova (1975), Jepps (1975). Also non-axisymmetric fields were considered; see Stix (1971), Krause (1971), Roberts and Stix (1972). It has been proved by Levy (1972) that dynamos of the $\alpha \omega$-type are also able to generate stationary fields; see also Stix (1973) and Deinzer et al. (1973, 1974).

Next we consider the electromotive force described by $\mu \beta_{1} \hat{\boldsymbol{\omega}} \times \overline{\mathbf{j}}$, i.e. the $\omega \times \mathbf{j}-$ effect. In combination with differential rotation this effect too allows dynamos. In 
other words, differential rotation and a special type of anisotropic electric conductivity can lead to dynamos. In the first investigations of models of that kind carried out by Rädler (1969c, 1970) and by Roberts (1972) only stationary fields were found. Dealing with the possibility of alternating fields Roberts (1972) got numerical results which suggest that such fields exist too, but because of some convergency difficulties he regarded these results as not quite convincing. Recent results by Rädler (1975) confirm that alternating fields can occur. Some details will be given in the appendix.

As the last of the electromotive forces listed in (3.1) we consider that described by $\boldsymbol{\mu} \boldsymbol{\alpha}_{9}(\hat{\mathbf{g}} \cdot \hat{\boldsymbol{\omega}}) \hat{\mathbf{g}} \times \overline{\mathbf{j}}$. Since it is very similar to $\mu \boldsymbol{\beta}_{1} \hat{\mathbf{\omega}} \times \overline{\mathbf{j}}$ we have good reason to assume that it together with differential rotation can give rise to dynamos too.

\subsection{REMARKS CONCERNING THE FURTHER ELABORATION OF MODELS OF THE SOLAR CYCLE}

Many efforts have been devoted to the elaboration of dynamo models for the solar cycle which reflect as many features of observations as possible. Suppose the basis is correct, one may, when fitting the models to observations, draw some conclusions on parameters inaccessible for direct observations, e.g. on the radial dependence of angular velocity or on parameters characterizing the fluctuating motions.

In this context we must have in mind that each of the electromotive forces occurring in Ohm's law (2.37) can influence the excitation conditions and the space and time behaviour of the magnetic fields. We have to scrutinize carefully which of these electromotive forces should be taken into account and which of them could be neglected. In most of the hitherto discussed dynamo models for the solar cycle only the idealized $\alpha$-effect and the turbulent conductivity were involved, i.e. only effects which are already known from the case of homogeneous isotropic turbulence, and all other effects of turbulence were cancelled. The possible influence of these effects should be discussed in more detail, otherwise the conclusions of the kind mentioned above are only of restricted value.

Considering again the generation of the poloidal from the toroidal field we have to clarify which of the electromotive forces listed in (3.1) should be taken into account. It seems that the $\alpha$-effect plays the most important part indeed. As for orders of magnitude, the ratio of the idealized $\alpha$-effect to the $\omega \times \mathbf{j}$-effect is given by $\alpha_{1} \lambda_{B} / \beta_{1}$, where $\lambda_{B}$ is a characteristic length for the variation of the magnetic field. Provided (2.35a) and (2.39) determine the orders of magnetic of $\alpha_{1}$ and $\beta_{1}$ even when only $h$ is involved and $l_{1}$ and $m_{1}$ are cancelled, this ratio can be replaced by $\lambda_{B} / \lambda_{K}$ with $\lambda_{K}$ as given in (2.22). It is to be expected that $\lambda_{B} / \lambda_{K} \gg 1$; for according to the abovementioned estimates $\lambda_{K}$ is hardly bigger than $10^{4} \mathrm{~m}$. In this way the $\omega \times j$-effect turns out to be of minor importance, and the same can be concluded for the related effect proportional to $(\hat{\mathbf{g}} \cdot \hat{\boldsymbol{\omega}}) \hat{\mathbf{g}} \times \overline{\mathbf{j}}$. These arguments, however, should undergo a more detailed examination. Some investigations of dynamo models in which beside the differential rotation both the $\alpha$-effect and the $\omega \times \mathbf{j}$-effect are included have been carried out by Rädler (1975).

The behaviour of the toroidal field can be influenced by some of the electromotive forces occurring in (2.37) even without the assistance of the poloidal field. Using 
(2.25) we write these electromotive forces in the form

$$
-\gamma \hat{\mathbf{g}} \times \overline{\mathbf{B}}, \quad-\mu\left(\gamma_{1}-\gamma_{2}\right) \hat{\mathbf{g}}(\hat{\mathbf{g}} \cdot \overline{\mathbf{j}}), \quad-\mu \gamma_{2} \overline{\mathbf{j}} .
$$

The first of them, which can act in the same way as a diamagnetism of the matter, can strongly influence the dynamo mechanism. This becomes clear from the dynamo models investigated by Ruzmaikin and Ivanova (1975) in which diamagnetism is included, even though in a manner which does not correspond exactly to the conception of mean-field electrodynamics outlined here. The last two electromotive forces in (3.2), which can be interpreted in terms of an anisotropy of the turbulent conductivity, have not been investigated in this respect up to now.

Finally we have to consider the generation of the toroidal from the poloidal field and that influence on the poloidal field which occurs without the assistance of the toroidal one. All the electromotive forces due to turbulence which have to be discussed here, are induced by the poloidal field. As long as the poloidal is small compared with the toroidal field, they should be small compared with those given in (3.1) and (3.2). Therefore, they are presumably of minor importance for dynamo models as considered here.

As indicated above, our considerations are restricted to axisymmetric magnetic fields. If non-axisymmetric ones should be included some points have to be rediscussed.

\section{The Influence of the Mean Magnetic Field on the Motions and its Consequences for the Dynamo Mechanism}

\subsection{BASIC IDEAS}

Up to now we have dealt with the question of how mean electromagnetic fields in electrically conducting matter are influenced by the motions of the matter but we did not pay attention to the fact that the motions can be influenced by the electromagnetic fields. Within the frame of such considerations, dynamo mechanisms turn out to be possible which allow the mean magnetic fields to grow incessantly. In accordance with that, the kinematic dynamo theory of the solar cycle allows arbitrary mean-field amplitudes. However, if a magnetic field grows we have to expect that also its back reaction to the motions due to Lorentz forces becomes stronger and, finally, provides for a limitation of the field strength. In this way also the mean-field amplitude in the solar cycle is controlled.

Already this point gives rise to extend the foregoing considerations so that this influence of the electromagnetic fields on the motions is included. Doing so we overstep the bounds of mean-field electrodynamics and are confronted with much more complicated aspects of mean-field magnetohydrodynamics. Only some of the problems arising here have been tackled up to now.

To begin with, we have to complete the basic electrodynamic Equations (2.1) by hydrodynamic equations. We want to demonstrate only some basic features. For this purpose we restrict ourselves to incompressible media. Then only the Navier-Stokes 
equation and the continuity equation have to be added which read

$$
\begin{aligned}
& \rho\left(\frac{\partial \mathbf{u}}{\partial t}+(\mathbf{u} \cdot \operatorname{grad}) \mathbf{u}\right)=-\operatorname{grad} p+\rho \mathbf{f}+\mathbf{j} \times \mathbf{B} \\
& \operatorname{div} \mathbf{u}=0 .
\end{aligned}
$$

Here $\rho$ is the mass density, which is to be regarded as a constant, $p$ is the hydrodynamic pressure, and $\mathbf{f}$ denotes viscous or other body forces. Finally, $\mathbf{j} \times \mathbf{B}$ represents the Lorentz force which is responsible for the influence of the electromagnetic fields on the motion. In the same way as with the Equations (2.1) we take the average of the Equations (4.1) thus obtaining

$$
\begin{aligned}
& \rho\left(\frac{\partial \overline{\mathbf{u}}}{\partial t}+(\overline{\mathbf{u}} \cdot \operatorname{grad}) \overline{\mathbf{u}}\right)=-\operatorname{grad} \bar{p}+\rho \overline{\mathbf{f}}+\overline{\mathbf{j}} \times \overline{\mathbf{B}}-\rho \overline{\left(\mathbf{u}^{\prime} \cdot \operatorname{grad}\right) \mathbf{u}^{\prime}}+\overline{\mathbf{j}^{\prime} \times \mathbf{B}^{\prime}} \\
& \operatorname{div} \overline{\mathbf{u}}=0 .
\end{aligned}
$$

Just as observed with Ohm's law, the formal correspondence between the NavierStokes equation for the original and the mean fields is disturbed by additional forces, $-\rho\left(\overline{\left.\mathbf{u}^{\prime} \cdot \text { grad }\right) \mathbf{u}^{\prime}}\right.$ and $\overline{\mathbf{j}^{\prime} \times \mathbf{B}^{\prime}}$, appearing with the mean fields. The first of them is already known from the theory of hydrodynamic turbulence; it can be interpreted in terms of Reynolds stresses.

This consideration shows that within the mean-field magnetohydrodynamics of incompressible media in addition to the electromotive force, $\overline{\mathbf{u}^{\prime} \times \mathbf{B}^{\prime}}$, the two forces, $-\rho\left(\overline{\left.\mathbf{u}^{\prime} \cdot \operatorname{grad}\right) \mathbf{u}^{\prime}}\right.$ and $\overline{\mathbf{j}^{\prime} \times \mathbf{B}^{\prime}}$, have to be investigated. As far as $\overline{\mathbf{u}^{\prime} \times \mathbf{B}^{\prime}}$ is concerned all general relations discussed above hold also when $\overline{\mathbf{u}}$ and $\mathbf{u}^{\prime}$ and, consequently, $K_{i j}$ are specified to depend on $\overline{\mathbf{B}}$. Of course, then $\overline{\mathbf{u}^{\prime} \times \mathbf{B}^{\prime}}$ is no longer linear in $\overline{\mathbf{B}}$. A part of the methods by which $\mathbf{u}^{\prime} \times \mathbf{B}^{\prime}$ was determined can be used also to determine $-\rho\left(\overline{\left.\mathbf{u}^{\prime} \cdot \operatorname{grad}\right) \mathbf{u}^{\prime}}\right.$ and $\overline{\mathbf{j}^{\prime} \times \mathbf{B}^{\prime}}$.

If we remove the restriction to incompressible media, some details become more complicated; e.g. correlations between $\rho^{\prime}$ and $\mathbf{u}^{\prime}$ have to be considered.

\subsection{SOME SPECIAL RESULTS}

The dynamo models of the solar cycle are determined by assumptions on both the mean motion and the electromotive force caused by the fluctuating motions. Since there is no observational evidence for a substantial variation of the mean motion during the cycle it is reasonable to suppose $\overline{\mathbf{u}}$ to be independent of $\overline{\mathbf{B}}$. There are, however, several reasons to assume that the fluctuating motions are considerably influenced by the magnetic field, so that for the electromotive force, $\overline{\mathbf{u}^{\prime} \times \mathbf{B}^{\prime}}$, a non-linear dependence on $\overline{\mathbf{B}}$ has to be expected.

There are several investigations of $\mathbf{u}^{\prime} \times \mathbf{B}^{\prime}$ in which this non-linear dependence on $\overline{\mathbf{B}}$ is considered. Unfortunately, in most cases suppositions were used which scarcely apply to the solar convection zone; nevertheless, the results may be suggestive here too.

Especially the $\alpha$-effect was considered which occurs with an originally homogeneous isotropic non-mirror-symmetric turbulence under the influence of a homogeneous mean magnetic field. As long as this influence is weak one may easily conclude 
that $\alpha$, now understood as a function of $\bar{B}$, has the form

$$
\alpha=\alpha_{0}\left(1-a \bar{B}^{2}\right)
$$

with some coefficients $\alpha_{0}$ and $a$; see Krause and Rädler (1971). Using second order correlation approximation and supposing $R_{m} \ll 1$ as well as incompressibility of the medium, Rüdiger (1974) computed the value of $a$. Within the same frame he discussed $\alpha$ for large $\bar{B}$ too and found that it vanishes as $\bar{B}^{-3}$ if $\bar{B} \rightarrow \infty$. Interesting relations for $\alpha$ have been derived by Vainshtein and Zeldovich (1972).

Furthermore, $\overline{\mathbf{u}^{\prime} \times \mathbf{B}^{\prime}}$ has been studied in detail, especially with respect to its dependence on $\overline{\mathbf{B}}$, for a random superposition of inertial waves in a rotating fluid by Moffatt (1970b, 1972) and Soward (1975). Comprehensive calculations for an inhomogeneous turbulence in a rotating fluid have been carried out by Roberts and Soward (1975).

Finally, first investigations on the consequences of an $\alpha$-effect which is non-linear in $\overline{\mathbf{B}}$ for dynamo mechanisms of the $\alpha \omega$-type have been presented by Stix (1973) and Jepps (1975).

\section{Appendix to Section 3.1}

We give here an example of a dynamo model which works on the basis of differential rotation and the $\omega \times \mathbf{j}$-effect and allows alternating magnetic fields. The model consists of a sphere of conducting moving matter. The mean motion is supposed to be a differential rotation with an angular velocity, $\omega$, given by

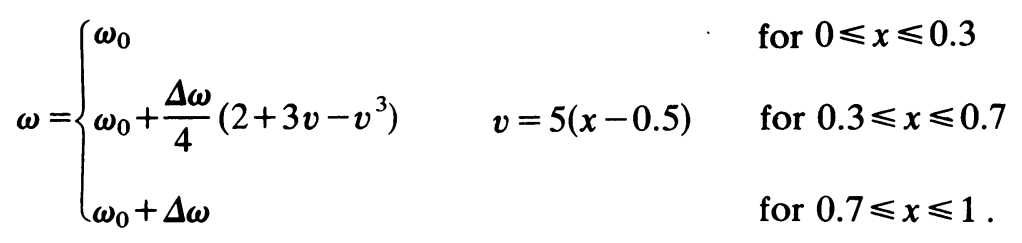

As for the electromotive forces induced by turbulence, only the $\omega \times \mathbf{j}$-effect is involved with a coefficient, $\beta_{1}$, specified by

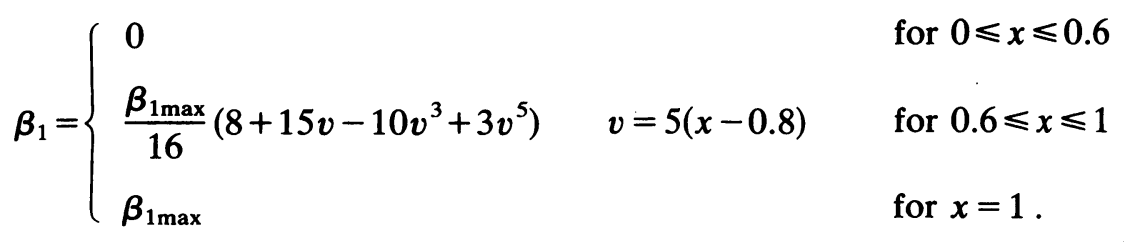

Here $\omega_{0}, \Delta \omega$ and $\beta_{1 \max }$ are constants, and $x$ is the normalized radius of the sphere with $x=1$ at its surface. Figure 2 shows the profiles of $\omega / \omega_{0}$ and $\beta_{1} / \beta_{1 \max }$. The surroundings of the sphere are supposed to be a vacuum. Again, only axisymmetric magnetic fields are considered. For the generation of the toroidal field from the 


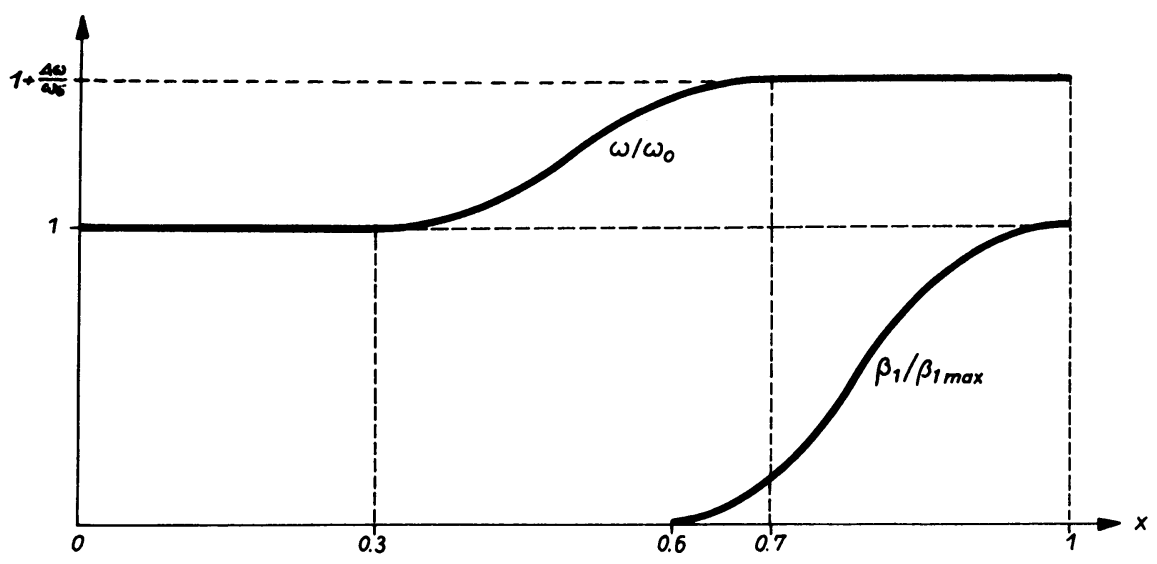

Fig. 2. Profiles of the normalized angular velocity, $\omega / \omega_{0}$, and the normalized $\omega \times \mathbf{j}$-coefficient, $\beta_{1} / \beta_{1 \max }$.

poloidal one only the differential rotation is taken into account, and the $\omega \times \mathbf{j}$-effect is neglected. The generation of the poloidal field from the toroidal one is, however, due to the $\omega \times \mathbf{j}$-effect. As one can easily see from the equations governing this model, the condition of excitation of magnetic fields can be expressed by a dynamo number, $P$, given by

$$
P=\mu^{2} \sigma^{2} \Delta \omega \beta_{1 \max } R^{2}
$$

where $R$ is the radius of the sphere. Since $\beta_{1 \max }$ may be supposed to be positive, $P>0$ corresponds to $\Delta \omega>0$, i.e. an inward decreasing angular velocity, and $P<0$ corresponds to $\Delta \omega<0$, i.e. an inward increasing angular velocity. Furthermore, a dimensionless frequency, $\Omega$, of the mean fields is used for which the time is measured in units of $\mu \sigma R^{2}$.

By means of a representation of the magnetic field by spherical harmonics the partial differential equations governing this model were reduced to an infinite set of ordinary differential equations. But only the first harmonics up to a certain order, say $N$, were taken into account, and the solutions of the corresponding truncated sets of equations were computed numerically for different $N$. In the case of convergency of these solutions for growing $N$, the solutions for sufficiently high $N$ were regarded as such of the original equations.

TABLE I

Marginal dynamo numbers, $P$, dimensionless frequencies, $\Omega$, and the types of the respective solutions

\begin{tabular}{rrl}
\hline $\boldsymbol{P}$ & $\boldsymbol{\Omega}$ & Type of solution \\
\hline-2347 & 16.6 & dipole \\
-2216 & 6.5 & quadrupole \\
+4914 & 35.5 & quadrupole \\
+19268 & 73.4 & dipole \\
\hline
\end{tabular}


For both $P>0$ and $P<0$ solutions were found which correspond to magnetic fields of dipole as well as of quadrupole type. In Table I the marginal values of $\boldsymbol{P}$ which were found within the region $-2500 \ldots+20000$ and the respective values of $\Omega$ were listed. Table II shows one example illustrating the convergency of the solutions of the truncated equations.

\section{TABLE II}

\begin{tabular}{l}
$\begin{array}{l}\text { Marginal dynamo numbers, } P \text {, and dimen- } \\
\text { sionless frequencies, } \Omega \text {, for related solu- } \\
\text { tions of the truncated equations in depen- } \\
\text { dence on the truncation parameter, } N\end{array}$ \\
\hline$N$
\end{tabular}

\section{References}

Batchelor, G. K.: 1950, Proc. Roy. Soc. A201, 406.

Braginski, S. I.: 1964, Zh. Eksper. Theoret. Fiz. 47, 1084.

Csada, I. K.: 1951, Acta Phys. Hung. 1, 235.

Deinzer, W. and Stix, M.: 1971, Astron. Astrophys. 12, 111.

Deinzer, W., v. Kusserow, H.-U., and Stix, M.: 1973, Mitteilungen Astron. Ges. 34, 155.

Deinzer, W., v. Kusserow, H.-U., and Stix, M.: 1974, Astron. Astrophys. 36, 69.

Drobyshevski, E. M. and Yuferev, V. S.: 1974, J. Fluid Mech. 65, 33.

Jepps, S. A.: 1975, J. Fluid. Mech. 67, 625.

Kasantsev, A. P.: 1967, Zh. Eksper. Theoret. Fiz. 53, 1806.

Krause, F.: 1967, Habilitationsschrift Univ. Jena.*

Krause, F.: 1971, Astron. Nachr. 293, 187.

Krause, F. and Rädler, K.-H.: 1971, in Handbuch der Plasmaphysik und Gaselektronik 2 (ed. by R. Rompe and M. Steenbeck), Akademie-Verlag, Berlin.

Krause, F. and Roberts, P. H.: 1973a, Astrophys. J. 181, 977.

Krause, F. and Roberts, P. H.: 1973b, Mathematika 20, 24.

Krause, F.: 1975, this volume, p. 305.

Krause, F. and Rüdiger, G.: 1975, Solar Phys. 42, 107.

Levy, E. H.: 1972, Astrophys. J. 171, 621.

Moffatt, H. K.: 1970a, J. Fluid Mech. 41, 435.

Moffatt, H. K.: 1970b, J. Fluid Mech. 44, 705.

Moffatt, H. K.: 1972, J. Fluid. Mech. 53, 385.

Parker, E. N.: 1955, Astrophys. J. 122, 293.

Rädler, K.-H.: 1966, Dissertation Univ. Jena.

Rädler, K.-H.: 1968a, Z. Naturforsch. 23a, 1841.*

Rädler, K.-H.: 1968b, Z. Naturforsch. 23a, 1851.*

Rädler, K.-H.: 1969a, Geodät. Geophys. Veröffentl. Potsdam, Reihe II, Heft 13, 131.

Rädler, K.-H.: 1969b, Monatsber. Dtsch. Akad. Wissensch. Berlin 11, 195.*

Rädler, K.-H.: 1969c, Monatsber. Dtsch. Akad. Wissensch. Berlin 11, 272.*

Rädler, K.-H.: 1970, Monatsber. Dtsch. Akad. Wissensch. Berlin 12, 468.

Rädler, K.-H.: 1974, Astron. Nachr. 295, 85.

Rädler, K.-H.: 1975, Astron. Nachr. (in preparation).

Roberts, P. H.: 1971, in W. H. Reid (ed.), Mathematical Problems in the Geophysical Sciences, American Mathematical Society, Providence, R. I., p. 129. 
Roberts, P. H.: 1972, Phil. Trans. Roy. Soc. London A272, 663.

Roberts, P. H. and Stix, M.: 1971, 'The Turbulent Dynamo', NCAR Technical Note TN/IA-60.

Roberts, P. H. and Stix, M.: 1972, Astron. Astrophys. 18, 453.

Roberts, P. H. and Soward, A. M.: 1975, Astron. Nachr. 296, 49.

Rüdiger, G.: 1974, Astron. Nachr. 295, 275.

Ruzmaikin, A. and Ivanova, B.: 1975, Astron. Zh. (in print).

Soward, A. M.: 1975, J. Fluid Mech. 69, 145.

Steenbeck, M. and Krause, F.: 1969, Astron. Nachr. 291, 49.

Steenbeck, M., Krause, F. and Rädler, K.-H.: 1963, Sitzungsber. Dtsch. Akad. Wiss. Berlin, Klasse Math.Phys.-Tech., Heft 1.

Steenbeck, M., Krause, F., and Rädler, K.-H.: 1966, Z. Naturforsch. 21a, 369.*

Stix, M.: 1971, Astron. Astrophys. 13, 203.

Stix, M.: 1973, Astron. Astrophys. 24, 275.

Stix, M.: 1974, Astron. Astrophys. 37, 121.

Stix, M.: 1975, this volume, p. 367.

Sweet, P. A.: 1950, Monthly Notices Roy. Astron. Soc. 110, 69.

Vainshtein, S. I. and Zeldovich, Ja. B.: 1972, Usp. Fiz. Nauk 106, 431 (Soviet Phys.-Usp. 15, 159 (1972)).

Articles marked with an asterisk have been translated into English by Roberts and Stix (1971).

\section{DISCUSSION}

Vainshtein: There is $\alpha$-effect without rotation caused only by magnetic forces. It is a nonlinear effect. I think that you must take it into account. In this case $\alpha \sim \mathbf{B} \cdot$ curl B.

Krause: In a paper by Roberts and Soward (Roberts, P. H. and Soward, A. M.: 1975, Astron. Nachr. 296) it was shown that in the first approximation no $\alpha$-effect is caused by the helicity of a mean magnetic field. More detailed, writing down $\alpha=a \cdot(\overline{\mathbf{B}} \cdot \operatorname{curl} \overline{\mathbf{B}})$, the factor $a$ will prove to be zero in the first approximation in $\overline{\mathbf{B}}$. I checked this result to be correct. My question is, in what approximation you found the constant $a$ to be unequal zero.

Vainshtein: The term (B - curl B) appears in the first nonlinear approximation.

Stix: If the coefficients of the various electromotive forces are difficult to obtain, can you at least obtain information about the sign of the coefficients? In particular what is the sign of $\beta_{1}$, the coefficient of the $\boldsymbol{\omega} \times \mathbf{j}$ term?

Rädler: In all calculations I know the coefficient $\beta_{1}$ turned out to be positive. For a certain range of suppositions, departing from Bochner's theorem, it can be shown that $\beta_{1}$ must necessarily be positive.

\section{Notes added in proof concerning chapter 3}

Strictly speaking, the factor $\alpha_{1}$ in (3.1) has to be replaced by $\alpha_{1}-\left(\alpha_{9}+\alpha_{10}\right) / r$, and $\gamma$ in (3.2) by $\gamma-\left(\gamma_{2}+\gamma_{3}\right) / r$, where $r$ is the radial coordinate. With respect to the foregoing discussions, however, this is of minor importance.

The above-mentioned estimation of the ratio $\alpha_{1} \lambda_{B} / \beta_{1}$ implies a special assumption on $h$. Obviously, $\alpha_{1}$ depends only on the symmetric and $\beta_{1}$ only on the antisymmetric part of $h$ where symmetry with respect to $(\hat{\omega} \cdot \xi)$ is considered. Only if both parts are of the same order of magnitude $\alpha_{1} \lambda_{B} / \beta_{1}$ may be replaced by $\lambda_{B} / \lambda_{K}$. However, this assumption and, consequently, the conclusion on the minor importance of the $\omega \times \mathbf{j}$-effect are questionable. 